\title{
Commercially available kits for manual and automatic extraction of nucleic acids from formalin-fixed, paraffin-embedded (FFPE) tissues
}

\author{
Boštjan J. Kocjan ${ }^{1}$, Lea Hošnjak ${ }^{1}$, Mario Poljak ${ }^{1 凶}$
}

\begin{abstract}
Introduction: Formalin-fixed, paraffin-embedded (FFPE) tissues represent an invaluable source for diagnostic purposes when fresh clinical material is unavailable, and also for molecular and epidemiological studies. The recovery of nucleic acids from FFPE tissues is particularly challenging, and several in-house methods have been developed for this purpose over the last three decades. Recently, several commercial kits specifically developed for DNA and/or RNA extraction from FFPE tissues have been introduced to the market, but their inventory is not available in peer-reviewed literature.

Methods: This article provides the first comprehensive inventory of commercial FFPE DNA/RNA extraction kits currently available on the market and describes their basic characteristics and features.

Results: A total of 69 commercial kits from 43 companies were identified. Thirty-five kits were developed specifically for DNA extraction, 22 for RNA extraction, and 12 for both DNA and RNA extraction. Only two commercial kits allow full automation of the entire nucleic acid extraction procedure. The tissue deparaffinization step is omitted in many protocols by melting paraffin directly in a tissue lysis buffer. Purification of the released nucleic acids is mainly based on silica or resin adsorption technology. A formalin reverse cross-linking step to increase the quality of extracted DNA and RNA is an intrinsic part of over half of the kits identified. Conclusions: It is hope that this comprehensive list of available commercial kits for extracting nucleic acids from FFPE will encourage researchers to strongly consider using them in diagnostic and research settings instead of old-fashioned, crude, and probably less effective in-house methods.
\end{abstract}

Keywords: archival tissues specimens, formalin-fixed, paraffin-embedded tissue, FFPE, nucleic acid extraction, DNA, RNA

Received: 10 August 2015 | Returned for modification: 26 August 2015| Accepted: 2 September 2015

\section{Introduction}

Formalin-fixed, paraffin-embedded (FFPE) tissues stored in pathology departments worldwide represent an invaluable source for diagnostic purposes when fresh clinical material is unavailable and also for molecular and epidemiological studies. However, working with nucleic acids extracted from FFPE tissue specimens is particularly challenging due to cross-linking of bio-molecules and fragmentation of nucleic acids. Several factors affect the quality of nucleic acids obtained from FFPE tissues, most notably the $\mathrm{pH}$ of the fixative, the duration of tissue fixation, the age and storage conditions of FFPE tissue blocks, and the method used for their extraction (1). The integrity of DNA/RNA is generally affected by a multitude of these factors, generating a large diversity of sample quality and highly variable target amplification (2).

Finding a suitable method for extracting nucleic acids from a particular clinical specimen is a prerequisite for successful subsequent testing with molecular methods such as those based on polymerase chain reaction (PCR). During the last three decades, many specific approaches for extracting DNA/RNA from FFPE tissues, which is then used for PCR, have been reported. In the early 1990s, several protocols were developed for rapid extraction of DNA and/or RNA from FFPE specimens, including boiling FFPE tissue sections in chelating resin solution or distilled water $(3,4)$, incubation in sodium dodecyl sulfate (SDS) or alkali buffers combined with phenol/chloroform purification $(5,6)$, and sonication (7), all with varying degrees of success. Proteolytic treatment with proteinase $\mathrm{K}$ with or without subsequent organic solvent purification has been one of the most frequently used methods for DNA/
RNA extraction from FFPE specimens, generally resulting in a satisfactory DNA/RNA yield and integrity for subsequent molecular analyses (1). Introduction of silica adsorption technology in 1996 (8) has greatly revolutionized purification of nucleic acids; for example, by improving the purity of DNA/RNA molecules, reducing preparation times, eliminating the need for toxic chemicals, and making it possible to automate the entire procedure. Since then, several silica adsorption-based commercial kits have been developed for extracting DNA and/or RNA molecules from various fresh clinical specimens, including tissue, mucosal/skin swabs, blood, liquor, and various body fluids. Moreover, these particular kits (not originally developed for FFPE tissues) have also been frequently used for nucleic acid extraction from FFPE tissue specimens, some employing innovative modifications of the original extraction procedure, such as pretreatment of paraffin sections with elevated temperatures (9), melting of paraffin directly in tissue lysis buffers (10), and/or addition of a reverse formalin crosslinking step (10).

Several commercial kits specifically designed for nucleic acid extraction from FFPE tissue specimens have been recently introduced to the market and are gradually being used in research on FFPE $(11,12)$. To the best of our knowledge, an inventory of commercial kits specifically designed for nucleic acid extraction from FFPE is currently not available in peer-reviewed literature. Thus, this review provides the first comprehensive inventory of commercial manual and automatic FFPE DNA/RNA extraction kits and systems currently available on the market and describes their basic characteristics and features. 


\section{Methods}

The data for this review were retrieved through a detailed search of Medline/Pubmed, Web of Science, Scopus, Google Scholar, Google, and Bing between July 1 and July 30, 2015. In addition, official websites of companies manufacturing nucleic acid extraction kits were searched in detail. Despite our best efforts, due to rapid developments in FFPE nucleic acid extraction kits and a lack of corresponding peer-reviewed publications, it is likely that not all kits currently available on the global market were identified and the omission of any particular available kit is unintentional.

\section{Results}

\section{FFPE nucleic acid extraction kits}

As summarized in Table 1, we identified a total of 69 commercial kits specifically designed for nucleic acid extraction from FFPE tissue specimens from 43 companies that are currently available on the market. Of these, 35 kits were specifically developed for DNA extraction, 22 for RNA extraction, and 12 for both DNA and RNA extraction (Table 1). Some kits allow the recovery of RNA throughout a range of sizes, including smaller microRNAs (miRNAs) and small interfering RNAs (siRNAs). Fifty-one kits were designed for manual, mostly column-based DNA/RNA extraction, eleven for manual or automated extraction, five for automated extraction, and two for fully automated DNA/RNA extraction. Interestingly, the majority of kits identified were launched in the last few years, and with a few exceptions (e.g., the Qiagen QIAamp DNA FFPE Tissue Kit) they consequently lack documented performance evaluation in peer-reviewed literature.

In the majority of kits identified, the digestion of standardized amounts of FFPE tissue, measured in tissue sections of various thickness or milligrams, is performed in a tissue lysis buffer containing proteinase $\mathrm{K}$ (Table 1). Exceptions to these include the RealLine FFPET DNA Extraction Kit (Bioron Diagnostics, Ludwigshafen, Germany), Geno-Prep FFPE DNA Kit (Genolution Pharmaceuticals, Seoul, Korea), and TaKaRa DEXPAT Easy (TaKaRa, Shiga, Japan), for which tissue lysis is performed without enzyme digestion. Deparaffinization of FFPE tissue sections using xylene is still one of the most frequent recommendations. However, to eliminate the use of flammable and malodorous xylene or d-limonene (Hemo-De), some companies have developed special, presumably less toxic, chemicals, making possible fast and efficient solubilization, phase separation, and removal of paraffin, such as Q-solution (TrimGen, Sparks, MD, USA), Deparaffinization solution (Qiagen, Hilden, Germany), BiOstic Paraffin Removal Reagent (MO BIO Laboratories, Carlsbad, CA, USA), and Paraffin Dissolver A (Exiqon, Vedbaek, Denmark). Because deparaffinization is laborious and can result in severe tissue loss and consequently lower DNA/RNA yield $(9,10)$, this step was omitted in many protocols, allowing melting of paraffin directly in tissue lysis buffers. However, the usual recommendation in this case is to trim away excess paraffin during tissue sectioning prior to starting tissue lysis. An incubation step at elevated temperatures (e.g., $70-90{ }^{\circ} \mathrm{C}$ for various times) following tissue lysis to partially remove formalin cross-links of the released DNA/RNA, thus improving the quality and DNA/RNA performance in downstream assays $(1,13)$, was identified in 41 kits with available information.

Of the available manual kits, the recently launched FFPE DNA Extraction Kit (Roche Molecular Systems Inc., Alameda, CA, USA) allows extraction of DNA from FFPE tissues in two steps in only 48
67 minutes using inventory heat elution technology. FFPE tissue sections including paraffin are placed into a specially designed heat-elution column containing resin, which is first heated to 56 ${ }^{\circ} \mathrm{C}$ for 1 hour to lyse the tissue. Following tissue lysis, pressure is created in the column as the liquid is briefly incubated at $98{ }^{\circ} \mathrm{C}$, allowing the elution and purification of genomic material.

\section{Automation of extraction of nucleic acids from FFPE}

In comparison to the manual procedure, automated protocols may produce better nucleic acid extraction reproducibility, require less tissue input, and/or require less hands-on time $(14,15)$. As already mentioned, we identified 16 FFPE DNA/RNA kits that were developed to work with systems that allow automated extraction of nucleic acids (Table 1). In most of the cases, tissue digestion with proteinase $\mathrm{K}$ is performed in an external water bath or a rocking platform until the sample is completely lysed. The tissue digest without tissue debris is then manually transferred to a fully automated instrument containing ready-to-use reagents or cartridges with buffers optimized for one-step extraction of DNA and/or RNA, usually with the use of magnetized beads. Interestingly, two of the nucleic acid extraction systems identified have an integrated (combined) paraffin-melting and tissue-lysis step, thus allowing full automation of the entire nucleic acid extraction procedure (Table 1).

The first, the Siemens system (Siemens Healthcare Diagnostics, Tarrytown, NY, USA), employs an automated Tissue Preparation System (Hamilton MICROLAB STARlet IVD instrument) and the VERSANT Tissue Preparation Reagents kit with universal chemistry for simultaneous co-isolation of DNA and RNA from a single FFPE tissue section in a single step. This extraction system is based on iron oxide beads coated with a nanolayer of silica that are homogenous in shape and size (spherical, $<1 \mu \mathrm{m}$ ), which allows improved reproducibility, recovery, and quality of nucleic acids $(16,17)$. In the first step, simultaneous paraffin melting and FFPE tissue lysis are performed, followed by non-specific binding of tissue debris to silica beads under non-chaotropic conditions. Removal of the remaining undigested tissue is necessary to achieve effective and complete automation because it may interfere with accurate liquid handling and result in clogging pipette tips (17). A xylene-free deparaffinization step, based on hydrophobic absorption of molten paraffin into the inner polypropylene wall of the sample tube during the lysis process, further allows automation of the entire procedure (17). In the following step, the lysis fluid containing DNA/RNA is transferred to a chaotropic buffer containing fresh silica beads. Following binding and washing, pure DNA/RNA is eluted from silica beads and stored until downstream applications. The system is able to process a total of 48 FFPE samples (one or more $5-10 \mu \mathrm{m}$ thick FFPE tissue sections) in less than 4 hours, including a 30-minute incubation step for DNase I digestion if pure RNA is required (17).

The second system, the MagCore system (MagCore, Châtel-StDenis, Switzerland), employs an automated MagCore HF16 Automated DNA/RNA Purification System and Genomic DNA FFPE One-Step Kit and makes possible single-step extraction of total DNA from one to five FFPE tissue sections. In the first step, simultaneous paraffin melting and FFPE tissue lysis is performed, which is followed by DNA purification using cellulose-coated magnetic beads; this particular technology is characterized by high binding capacity and high purity of the nucleic acids obtained. The MagCore system is able to process up to 16 FFPE samples (up to $5 \mu \mathrm{m}$ thick FFPE tissue sections) in less than 70 minutes. 
Table 1 | List of commercially available kits for extracting DNA and/or RNA from FFPE tissue specimens. (continued on next page)

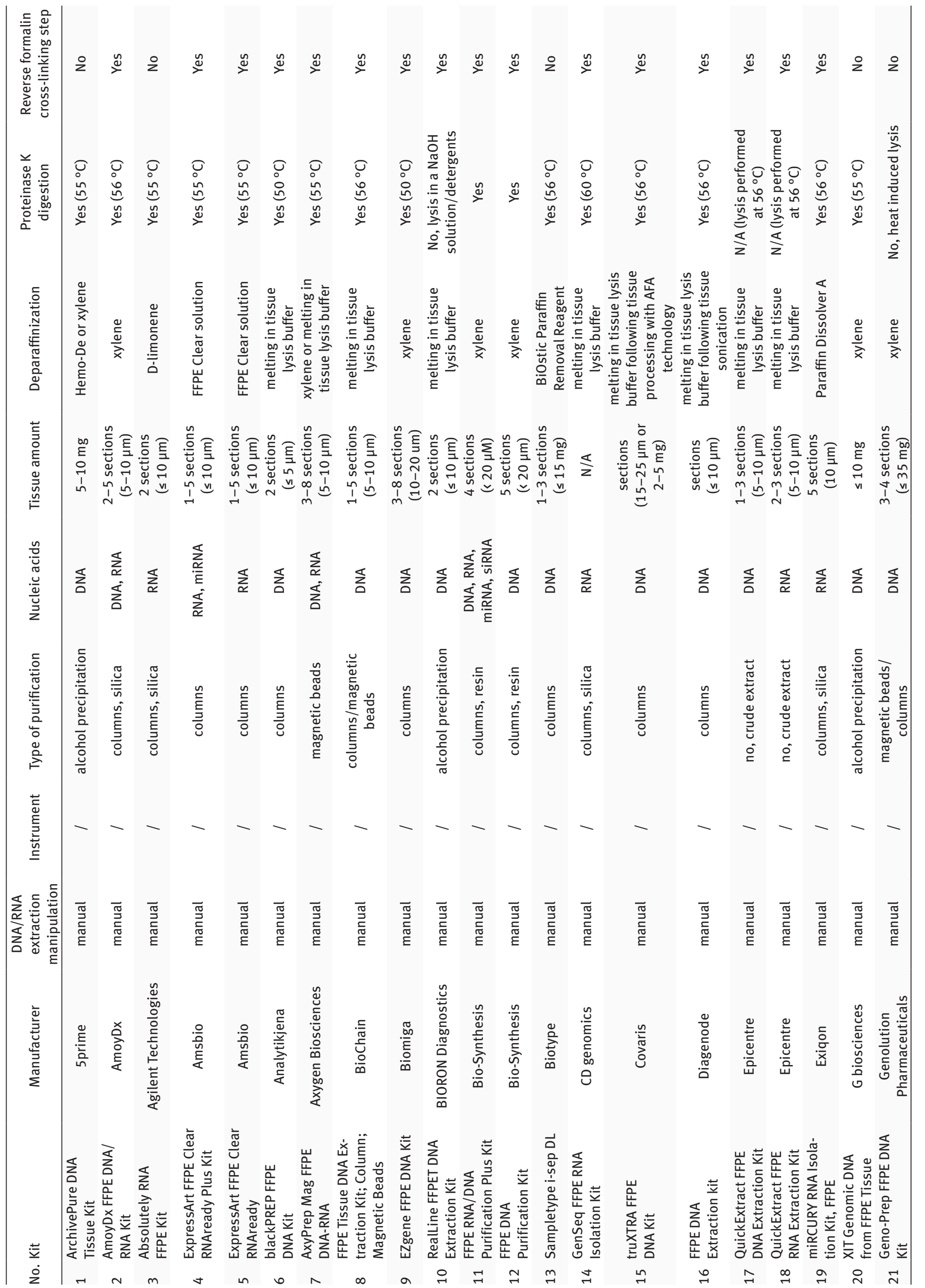




\section{Table 1 | Continued.}

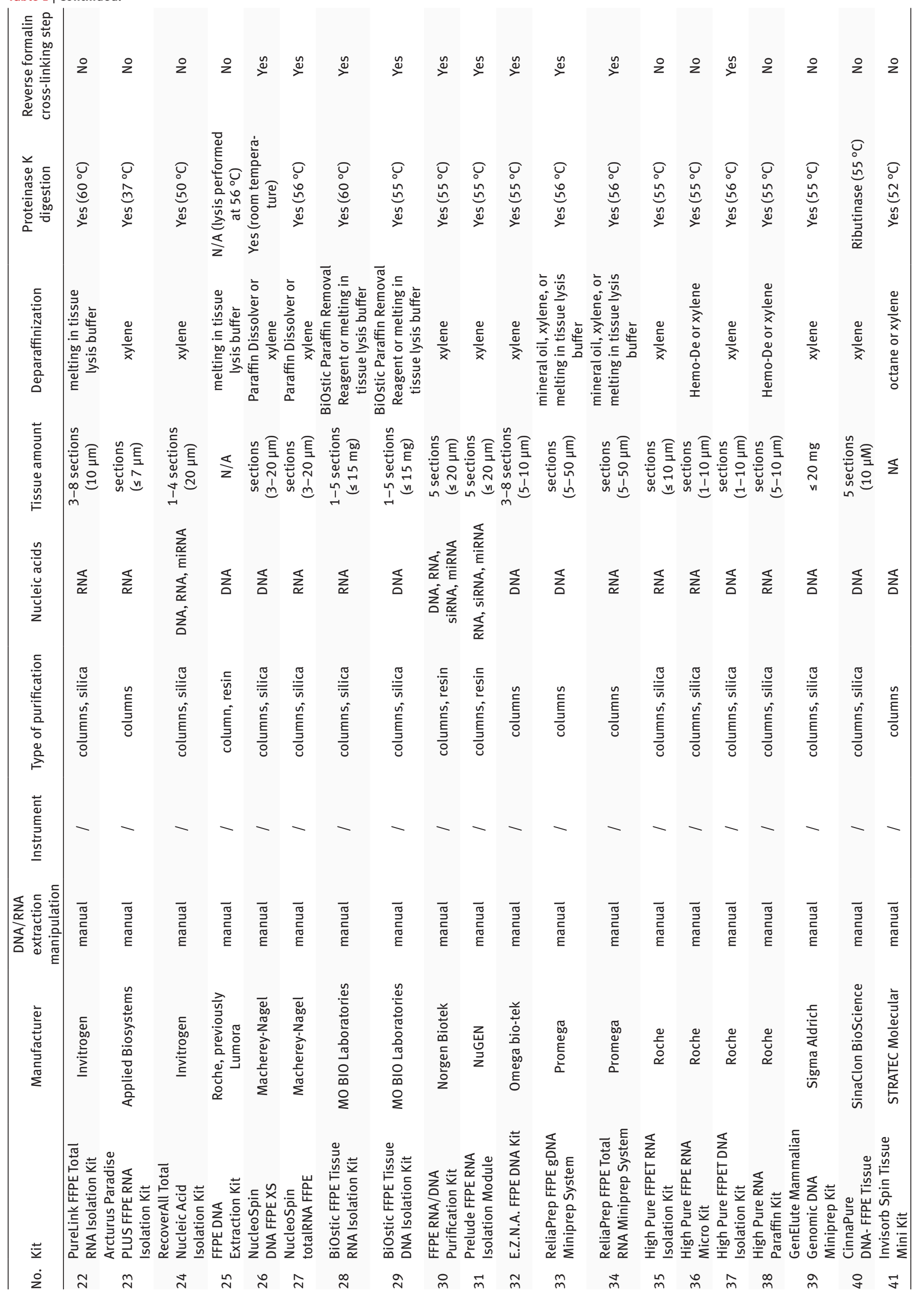




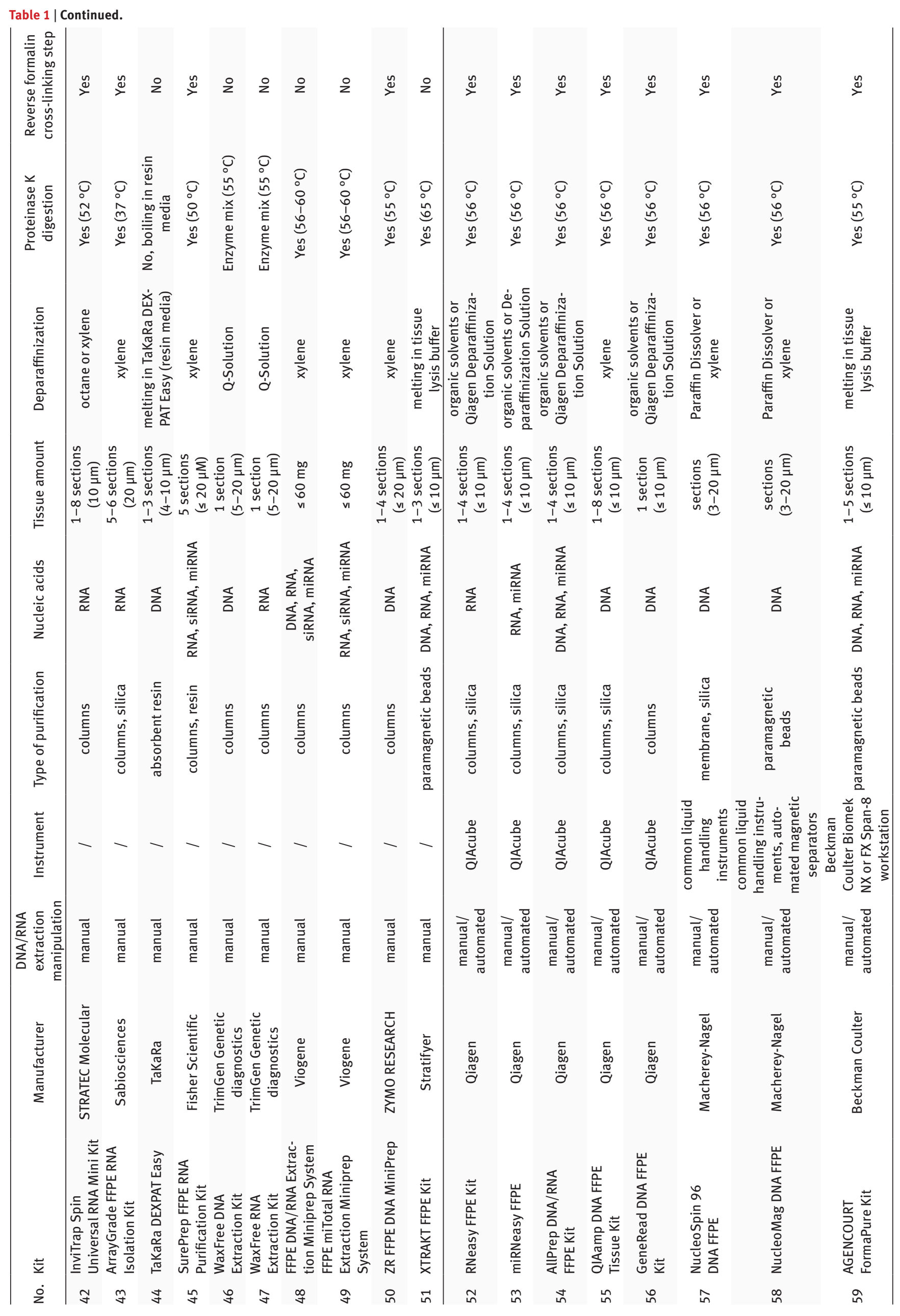




\section{Table 1 | Continued.}

\begin{tabular}{|c|c|c|c|c|c|c|c|c|c|c|}
\hline 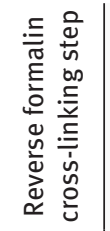 & $\stackrel{\varrho}{\rightleftharpoons}$ & $\stackrel{\varrho}{\rightleftharpoons}$ & $\stackrel{\mathscr{\Perp}}{=}$ & 운 & 울 & 울 & 운 & $\stackrel{\varrho}{\nu}$ & $\stackrel{\circ}{z}$ & 운 \\
\hline 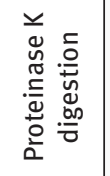 & $\begin{array}{l}0 \\
0 \\
0 \\
0 \\
0 \\
\stackrel{0}{0}\end{array}$ & 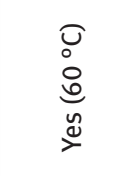 & 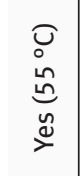 & $\begin{array}{l}0 \\
0 \\
0 \\
.0 \\
y \\
\searrow\end{array}$ & 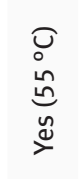 & 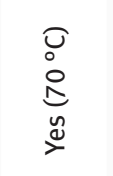 & $\begin{array}{l}0 \\
0 \\
0 \\
.0 \\
y \\
\stackrel{0}{\nu}\end{array}$ & $\begin{array}{l}0 \\
0 \\
0 \\
.0 \\
\tilde{y} \\
\nu\end{array}$ & 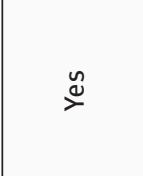 & $\begin{array}{l}0 \\
0 \\
i n \\
e \\
y \\
\searrow\end{array}$ \\
\hline 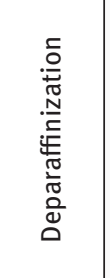 & 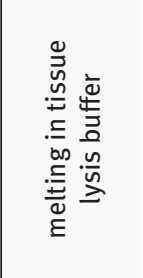 & 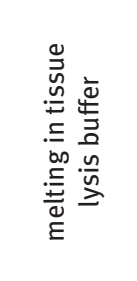 & 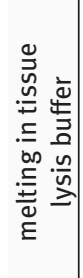 & 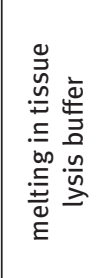 & $\frac{\stackrel{0}{\tilde{U}}}{\vec{x}}$ & 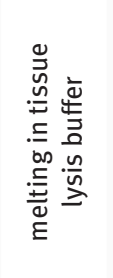 & 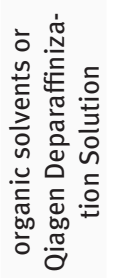 & 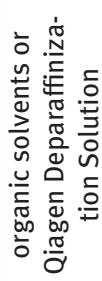 & 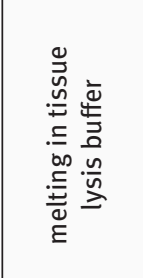 & 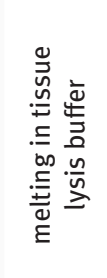 \\
\hline 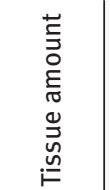 & 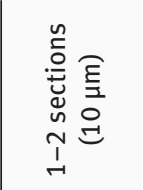 & 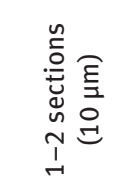 & 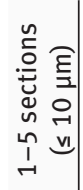 & 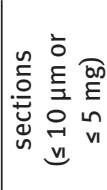 & 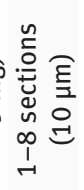 & 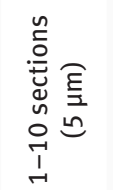 & 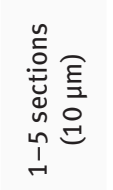 & 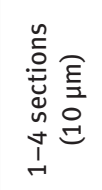 & 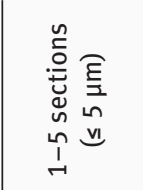 & $\frac{\pi}{z}$ \\
\hline 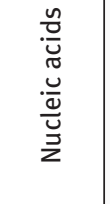 & \a & 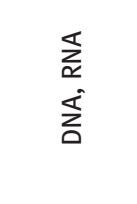 & 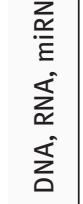 & 文 & $\sum_{0}^{1}$ & 文 & 壵 & $\sum_{0}^{\nwarrow}$ & \} $&{\text { 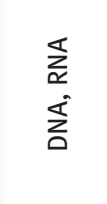 }} \\
{\hline \text { 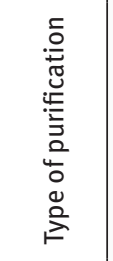 }} &{\text { 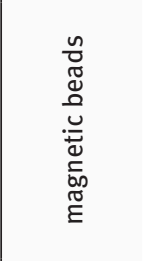 }} &{\text { 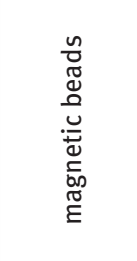 }} &{\text { 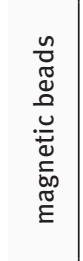 }} &{\text { 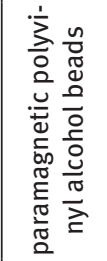 }} &{\text { 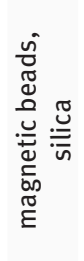 }} &{\text { 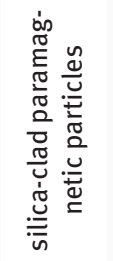 }} &{\text { 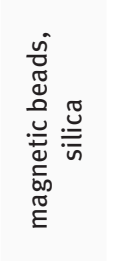 }} &{\text { 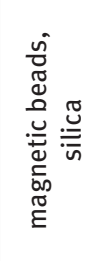 }} &{\text { 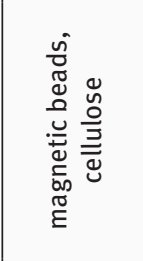 }} &{\text { 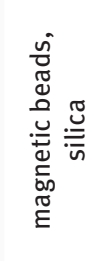 }} \\
{\hline \text { 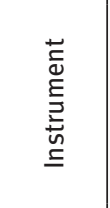 }} &{\text { 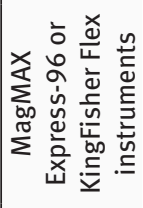 }} &{\text { 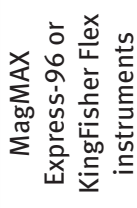 }} &{\Sigma} &{\text { 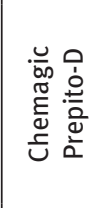 }} &{\text { 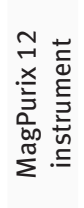 }} &{\text { 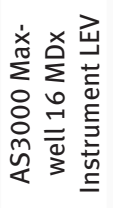 }} &{\text { 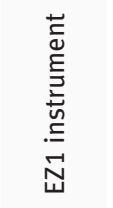 }} &{\text { 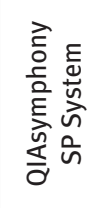 }} &{\text { 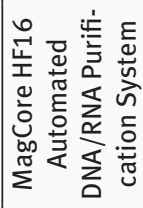 }} &{\text { 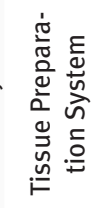 }} \\
{\hline \text { 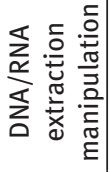 }} &{\text { 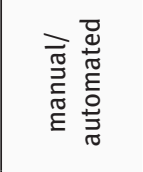 }} &{\text { 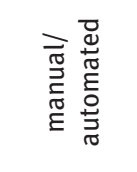 }} &{\text { 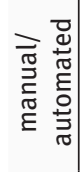 }} &{\text { 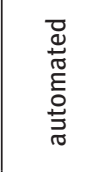 }} &{\text { 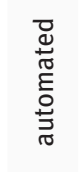 }} &{\text { 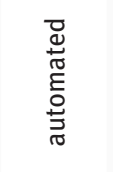 }} &{\text { 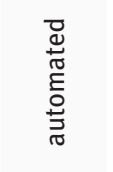 }} &{\text { 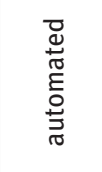 }} &{\text { 竞 }} &{\text { 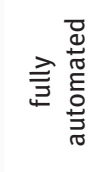 }} \\
{\hline \text { 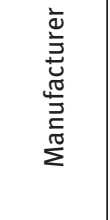 }} &{\text { 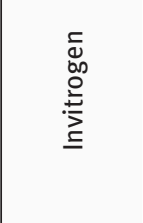 }} &{\text { 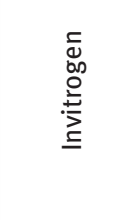 }} &{\text { 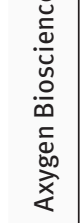 }} &{\text { 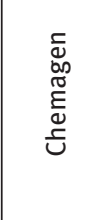 }} &{\text { 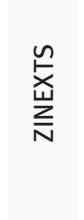 }} &{\text { 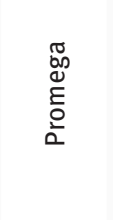 }} &{\text { 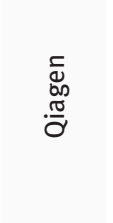 }} &{\text { 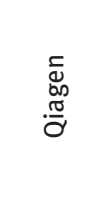 }} &{\text { 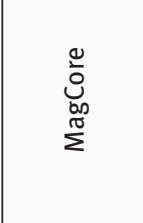 }} &{\begin{array}{c}n \\
\stackrel{n}{E} \\
\stackrel{E}{N} \\
i n\end{array}} \\
{\hline \text { 菏 }} &{\text { 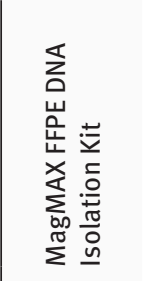 }} &{\text { 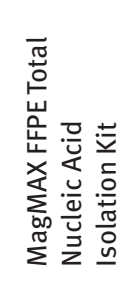 }} &{\text { 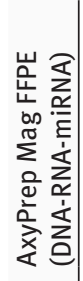 }} &{\text { 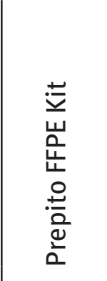 }} &{\text { 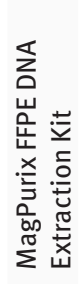 }} &{\text { 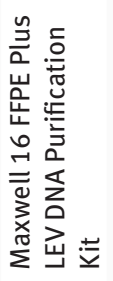 }} &{\text { 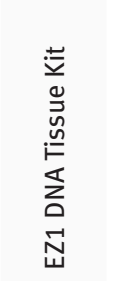 }} &{\text { 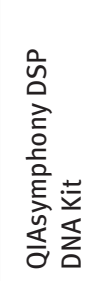 }} &{\text { 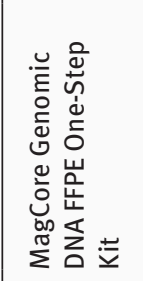 }} &{\text { 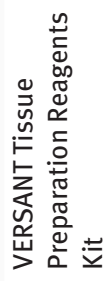 }} \\
{\hline \dot{0}} &{\text { ᄋ }} &{\vec{b}} &{\widetilde{\sigma}} &{\tilde{n}} &{\text { ఫర }} &{\hat{\sigma}} &{\bullet} &{\hat{\sigma}} &{\text { 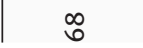 }} &{\text { aิ }} \\
$\hline
\end{tabular}




\section{Discussion}

Our inventory identified at least 69 commercial kits specifically developed for manual, automated, or fully automated extraction of nucleic acids from FFPE tissue specimens. The majority of commercial FFPE DNA/RNA kits employ proteolytic treatment with proteinase $\mathrm{K}$ to release nucleic acids from FFPE tissues. Purification of DNA/RNA molecules from lysis fluid is mostly based on silica or resin adsorption technology, although alcohol precipitation and cellulose-based purification are used as well. Many of the available kits allow removal of paraffin using special solubilisators or allow melting of paraffin directly in tissue lysis buffers, which can reduce the loss of tissue during the extraction procedure. An incubation step at an elevated temperature for partial removal of formalin cross-links of the released DNA/RNA is surprisingly used in more than half of the available kits. This particular treatment generally allows the release of longer fragments of nucleic acids, which might result in better performance in downstream assays.

Sixteen identified kits allow automated, walk-away purification of DNA/RNA from lysed FFPE tissues obtained through manual external preparations, which represents a major bottleneck for these methods and also their main drawback. Only two systems-the Siemens Tissue Preparation System/VERSANT Tissue Preparation Reagents kit and the MagCore HF16 Automated DNA/ RNA Purification System/MagCore Genomic DNA FFPE One-Step
Kit-have an integrated paraffin-melting/tissue-lysis step and therefore allow complete automation of nucleic acid extraction from FFPE tissues.

Because the majority of FFPE DNA/RNA extraction kits were launched in the last few years, they generally lack documented performance in peer-reviewed literature. However, recent headto-head comparison studies suggest that these kits might differ significantly in terms of DNA yield, purity, and quality $(12,18)$. Therefore, it seems that the transition to one of the available FFPE DNA/RNA commercial kits will not be so straightforward and will require extensive comparisons with the established lab protocol in advance. The final decision in choosing a particular kit will probably also depend on the price and required accompanying lab equipment.

Although we identified an abundance of commercial kits specifically developed for extraction of nucleic acids from FFPE tissue specimens, many researchers are still using rather old-fashioned, crude, and probably less effective in-house methods for extracting nucleic acids from FFPE. We hope that this inventory and the accompanying comprehensive list of available commercial kits will encourage researchers to strongly consider using them in diagnostic and research settings when dealing with FFPE tissue specimens, similar to what occurred during the last decade for the great majority of other clinical specimen types.

\section{References}

1. Bonin S, Stanta G. Nucleic acid extraction methods from fixed and paraffin-embedded tissues in cancer diagnostics. Expert Rev Mol Diagn. 2013;13:271-82.

2. Dietrich D, Uhl B, Sailer V, Holmes EE, Jung M, Meller S, et al. Improved PCR performance using template DNA from formalin-fixed and paraffin-embedded tissues by overcoming PCR inhibition. PLoS One. 2013;8:e77771.

3. Coates PJ, d'Ardenne AJ, Khan G, Kangro HO, Slavin G. Simplified procedures for applying the polymerase chain reaction to routinely fixed paraffin wax sections. J Clin Pathol. 1991;44:115-8.

4. Sepp R, Szabó I, Uda H, Sakamoto H. Rapid techniques for DNA extraction from routinely processed archival tissue for use in PCR. J Clin Pathol. 1994;47:318-23.

5. Jackson DP, Lewis FA, Taylor GR, Boylston AW, Quirke P. Tissue extraction of DNA and RNA and analysis by the polymerase chain reaction. J Clin Pathol. 1990;43:499-504.

6. Shi SR, Cote RJ, Wu L, Liu C, Datar R, Shi Y, et al. DNA extraction from archival formalin-fixed, paraffin-embedded tissue sections based on the antigen retrieval principle: heating under the influence of $\mathrm{pH}$. J Histochem Cytochem. 2002;50:1005-11.

7. Heller MJ, Robinson RA, Burgart LJ, TenEyck CJ, Wilke WW. DNA extraction by sonication: a comparison of fresh, frozen, and paraffin-embedded tissues extracted for use in polymerase chain reaction assays. Mod Pathol. 1992;5:203-6.

8. Melzak KA, Sherwood CS, Turner RFB, Haynes CA. Driving forces for DNA adsorption to silica in perchlorate solutions. J Colloid Interface Sci. 1996;181:635-44.

9. Steinau M, Patel SS, Unger ER. Efficient DNA extraction for HPV genotyping in formalin-fixed, paraffin-embedded tissues. J Mol Diagn. 2011;13:377-81.

10. Kocjan BJ, Maver PJ, Hosnjak L, Zidar N, Odar K, Gale N, et al. Comparative evaluation of the Abbott RealTime High Risk HPV test and INNO-LiPA HPV Genotyping Extra test for detecting and identifying human papillomaviruses in archival tissue specimens of head and neck cancers. Acta Dermatovenerol Alp Pannonica Adriat. 2012;21:73-5.
11. Jenko K, Kocjan B, Zidar N, Poljak M, Strojan P, Zargi M, et al. In inverted papillomas HPV more likely represents incidental colonization than an etiological factor. Virchows Arch. 2011;459:529-38.

12. Janecka A, Adamczyk A, Gasińska A. Comparison of eight commercially available kits for DNA extraction from formalin-fixed paraffin-embedded tissues. Anal Biochem. 2015;476:8-10.

13. Gilbert MT, Haselkorn T, Bunce M, Sanchez JJ, Lucas SB, Jewell LD, et al. The isolation of nucleic acids from fixed, paraffin-embedded tissues-which methods are useful when? PLoS One. 2007;2:e537.

14. Bohmann K, Hennig G, Rogel U, Poremba C, Mueller BM, Fritz P, et al. RNA extraction from archival formalin-fixed paraffin-embedded tissue: a comparison of manual, semiautomated, and fully automated purification methods. Clin Chem. 2009;55:1719-27.

15. van Eijk R, Stevens L, Morreau H, van Wezel T. Assessment of a fully automated high-throughput DNA extraction method from formalin-fixed, paraffin-embedded tissue for KRAS, and BRAF somatic mutation analysis. Exp Mol Pathol. 2013;94:121-5.

16. Hennig G, Gehrmann M, Stropp U, Brauch H, Fritz P, Eichelbaum M, et al. Automated extraction of DNA and RNA from a single formalin-fixed paraffin-embedded tissue section for analysis of both single-nucleotide polymorphisms and mRNA expression. Clin Chem. 2010;56:1845-53.

17. Henniq B. DNA/RNA extraction from FFPE tissue samples. Mater Methods. 2011;1:31.

18. Potluri K, Mahas A, Kent MN, Naik S, Markey M. Genomic DNA extraction methods using formalin-fixed paraffin-embedded tissue. Anal Biochem. 2015;486:17-23. 